\title{
Testing Nonthermal Plasma for Decontamination of Sensitive Weapon PlatForms ANd Systems
}

\section{June 2011}

\section{Prepared by}

Meng-Dawn Cheng

Distinguished R\&D Staff Member 


\section{DOCUMENT AVAILABILITY}

Reports produced after January 1, 1996, are generally available free via the U.S. Department of Energy (DOE) Information Bridge.

Web site http://www.osti.gov/bridge

Reports produced before January 1, 1996, may be purchased by members of the public from the following source.

National Technical Information Service

5285 Port Royal Road

Springfield, VA 22161

Telephone 703-605-6000 (1-800-553-6847)

TDD 703-487-4639

Fax 703-605-6900

E-mail info@ntis.gov

Web site http://www.ntis.gov/support/ordernowabout.htm

Reports are available to DOE employees, DOE contractors, Energy Technology Data Exchange (ETDE) representatives, and International Nuclear Information System (INIS) representatives from the following source.

Office of Scientific and Technical Information

P.O. Box 62

Oak Ridge, TN 37831

Telephone 865-576-8401

Fax 865-576-5728

E-mail reports@osti.gov

Web site http://www.osti.gov/contact.html

This report was prepared as an account of work sponsored by an agency of the United States Government. Neither the United States Government nor any agency thereof, nor any of their employees, makes any warranty, express or implied, or assumes any legal liability or responsibility for the accuracy, completeness, or usefulness of any information, apparatus, product, or process disclosed, or represents that its use would not infringe privately owned rights. Reference herein to any specific commercial product, process, or service by trade name, trademark, manufacturer, or otherwise, does not necessarily constitute or imply its endorsement, recommendation, or favoring by the United States Government or any agency thereof. The views and opinions of authors expressed herein do not necessarily state or reflect those of the United States Government or any agency thereof. 
Environmental Sciences Division

\title{
TESTING NONTHERMAL PLASMA FOR DECONTAMINATION OF SENSITIVE WEAPONS PLATFORMS AND SYSTEMS
}

Meng-Dawn Cheng, Ph.D.

June 2011

\author{
Prepared by \\ OAK RIDGE NATIONAL LABORATORY \\ Oak Ridge, Tennessee 37831-6038 \\ Managed by \\ UT-BATTELLE, LLC \\ For the \\ U.S. DEPARTMENT OF ENERGY \\ Under contract DE-AC05-000R22725
}




\section{Table of Contents}

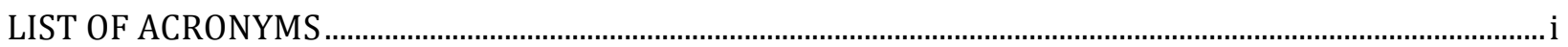

LIST OF FIGURES

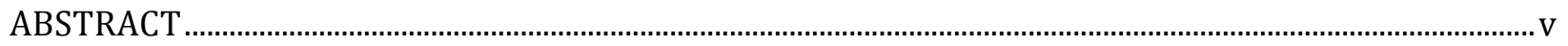

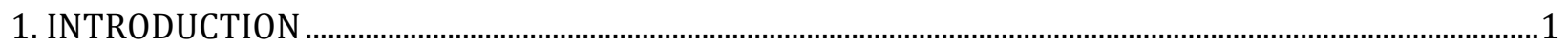

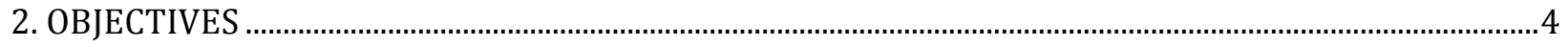

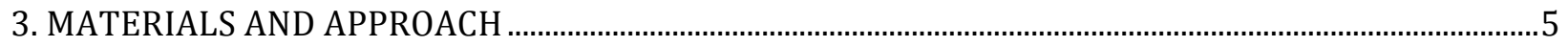

3.1. Description of Sample Substrate and Preparation of the Samples.......................................................5

3.2. Description of laboratory-scaled nonthermal plasma sources ….........................................................

3.3. Characterization of the material surfaces ..........................................................................................

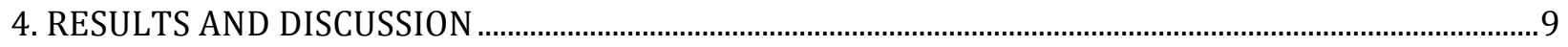

4.1. Effects of Composition of Carrier Gas Mixture on Removal .................................................................. 9

4.2. Evaluation of Surface Damage by Heat Associated with the Plasma ...................................................10

4.3. Removal of Oil Grease and CWA coated surfaces ................................................................................12

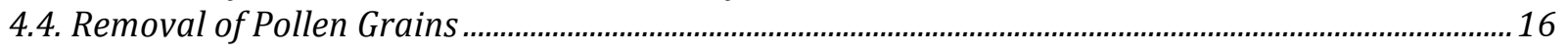

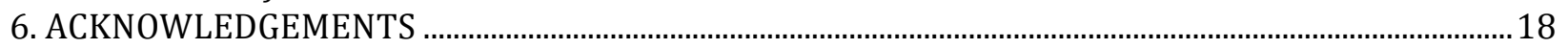

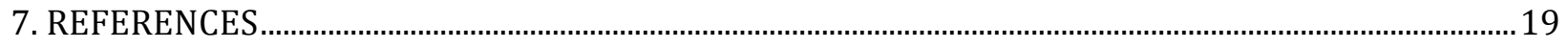

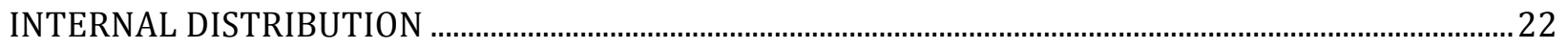

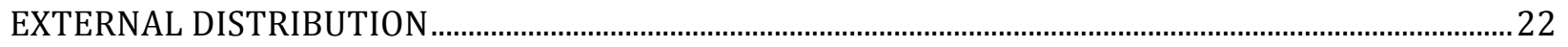




\section{LIST OF ACRONYMS}

3-D - Three dimensional

AP NTP - Atmospheric Pressure Nonthermal Plasma

CW - Continuous Wave

DC - Direct Current

DoD - Department of Defense

DOE - Department of Energy

$\mathrm{g} / \mathrm{L}$ - Gram per Liter

HAP - Hazardous Air Pollutants

He - Helium Molecule

$\mathrm{H}_{2}$ - Hydrogen Molecule

MA - State of Massachusetts

NC - State of North Carolina

NTP - Nonthermal Plasma

$\mathrm{O}_{2}$ - Oxygen Molecule

ORNL - Oak Ridge National Laboratory

OT - Optical Transmittance

$\mathrm{N}_{2}$ - Nitrogen Molecule

$\mathrm{RF}$ - Radio Frequency

SERDP - Strategic Environmental Research and Development Program

TIP - Triisopropyl Phosphate

VOC - Volatile Organic Compound

WI - State of Wisconsin 


\section{LIST OF FIGURES}

Figure 1a. Examples of AP NTP driven by radiofrequency power and shaped as a line

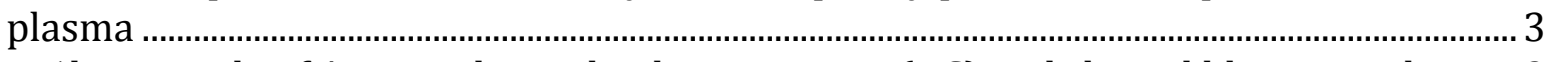

Figure 1b. Example of AP NTP driven by direct current (DC) and shaped like a round jet..... 3

Figure 2a. Photo showing an airframe sample used as the substrate in this project................. 5

Figure $2 \mathrm{~b}$. Photo showing the cross section of an airframe sample used as the substrate in this project. The honeycomb structure is sandwiched in between two layers of metallic-polymer skins in light blue color.

Figure 3. Photo of Experimental Setup of DC-NTP …………………………………………….... 7

Figure 4a. AP NTP from Surfx Technology ……………………………………………………..... 8

Figure 4b. Close up of the AP NTP Head ............................................................................................ 8

Figure 4c. Display of RF powered AP NTP on a airframe sample.................................................... 8

Figure 5. Plot of optical transmission verse time of exposure in minutes ................................. 10

Figure 6. A photo showing substrate under damage test ............................................................. 11

Figure 7. Removal of Oil Grease. [a] Untreated original substrate surface, [b] Oil treated surface of the same area as shown in [a], [c] RF-NTP treated surface of the same area

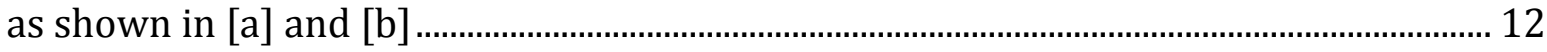

Figure 8. Setup for spectroscopic and aerosol detection.............................................................. 13

Figure 9. Size distribution of particles from RF-NTP treatment on oil layered airframe

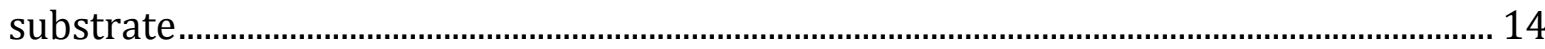

Figure 10. Spectrum of RF-NTP on CWA coated airframe substrate ........................................... 15

Figure 11a. Scanning Electron Microscopic Image of An Ambrosia Pollen Grain ..................... 16

Figure 11b. Image of Ambrosia Pollen Grains deposited on a glass slide.................................. 16

Figure 12. Raman Spectra of the Untreated Ambrosia Pollen Grains (0 min) and Treated after 3 , 4, and 10 min. ................................................................................................................... 17 


\section{THIS PAGE WAS INTENTIONALLY LEFT BLANK}


ORNL/TM-2011/155

\begin{abstract}
Efficient decontamination of military platforms and systems represents the first line of defense and protection for U.S. warfighters. Using solvents, wet decontamination approach generates secondary pollution and requires extra care of the contaminated solutions afterwards. The wet approach could also degrade material integrity of the treated platforms and systems. Alternative decontamination technique that does not erode the integrity and not produce secondary contamination is required. Nonthermal plasma was tested as a decontamination alternative for a number of pollutants in this project. A radiofrequency-powered nonthermal plasma technique was applied to biological aerosols, oil and grease, and paint-stained airframe structural material. Raman spectroscopy, scanning electron microscopy, and aerosol monitoring techniques were used to assess the effectiveness of the plasma decontamination technique. The nonthermal plasma technique was found to be useful in the cleanup of the stained surface, reduce the potency of the biological agents, and maintain the surface structure intact. Time-sequenced Raman analysis on biological aerosols indicates the antigens were either vaporized or decomposed during the treatment. Optical emission spectroscopy, aerosol electrometer, and scanning mobility particle sizer were used to monitor the nanoparticles and charges emissions during the treatment of oil and CWA-contaminated surface. ELISA assay shows the reduction and or elimination of the antigens, although scanning electron microscopy indicates the morphology of the biological agents remain unchanged by the plasma treatment. The technique does not produce secondary pollutants, is fairly safe to material and reasonably easy to operate. The technique is not a line-of-sight technique therefore it can be used to remove effectively organic contaminants and bioaerosols on a range of complex surfaces.
\end{abstract}




\section{INTRODUCTION}

Military weapons platforms and systems are exposed to a wide range of contaminants for various missions. Cleaning and decontamination are commonly performed by labor using a traditional cleaning agent that is "wet-based" such as bleaches like P-D-680 that is a petroleum-based solvent, which contains hazardous air pollutants and volatile organic compounds causing environmental and health concerns. Its successor, MIL-PRF-680 is also a petroleum-based solvent that contains the same amount of volatile organic compounds (VOCs, more than $750 \mathrm{~g} / \mathrm{L}$ ) but no hazardous air pollutants (HAPs). The aromatic content is approximately $1 \%$ by volume maximum, total phenol is no more than $0.5 \mathrm{ppm}$, dichlorobenzene is $0.5 \mathrm{mg} / \mathrm{L}$ maximum, and benzene is $0.5 \mathrm{mg} / \mathrm{L}$ max in MILPRF-680. Cold solvent cleaning of aircraft components is performed at organizational, intermediate, and depot level and usually takes the form of either spray sinks or batch loaded dip tanks. It is important to remember that the use of these solvents would release large amount of the synthetic organic compounds into the environment. The release could increase the risk of human exposure to the hazardous chemicals, particularly to the decontamination workers. Release of HAPs could also increase the atmospheric burden of organic compounds that might promote the formation of smog and degrade ambient air quality. Non-compliance of air quality in an airshed surrounding a military base or installation could cause undesirable consequences hindering training and other military activities.

MIL-PRF-680 solvents are specified for use in all maintenance facilities, impacting all the DoD, Coast Guard, and other activities. There are several alternatives to the MILPRF-680/P-D-680 solvents, including water-based and solvent-based cleaners. Waterbased cleaners contain detergents to remove grease and oil and may be used at elevated temperature and with various forms of agitation such as spraying or ultrasonics. Disadvantages include flash rusting, hydrogen embrittlement of high strength steel, and poor cleaning efficiency. Semi-aqueous processes incorporate not only detergents, but also solvents to improve effectiveness; some products contain solvents emulsified in water while others contain rinsable solvents.

Some regions in the United States allow limited amounts of exempt solvent in a solvent-cleaning blend. Air Pollution Control Districts in California and Maryland implement the most stringent requirements, usually stated in terms of VOC content and vapor pressure, respectively. The San Joaquin Valley Air Pollution Control District has imposed restrictions limiting the use of solvents with VOC content to a maximum of $25 \mathrm{~g} / \mathrm{L}$ in California. Maryland has forbidden the use of HAP-containing materials and has placed a limit of $1 \mathrm{mmHg}$ vapor pressure for degreasing solvents. This would require the acquisition of special solvent cleaning equipment by the military to keep using MIL-PRF-680 solvents that do not comply with this regulation.

There are other issues in addition to environmental protection and concerns over worker safety. For example, it is a major concern that the material integrity may be 
compromised after the use of aggressive chemical cleaning on large-frame aircraft construction materials. Material compatibility is critical for the follow-up use of cleaned or decontaminated weapons platforms and systems. The material compatibility problems directly affect the performance of the structural integrity of an aircraft frame, therefore, impacting the success of a mission. Previous tests at the Eglin Air Force Base in Florida using EasyDecon DF-100 on a BIS (2 ethylhexyl) hydrogen phosphite sprayed C-141 aircraft showed significant embrittlement of high strength steel. The failures were encountered in as little as 1.7 hours at $45 \%$ notched tensile strength. Thus, the results raised serious concerns about the use of water-based and non-aqueous liquid cleaning chemicals on aircraft construction materials. Furthermore, liquid waste produced by the cleaning process could potentially cause secondary contamination to soil and water in the environment. The liquid waste also increases the risk of worker exposure that could be avoided totally if a non-liquid cleaning technique can be developed.

One alternative to the "wet" approach is the Atmospheric Pressure NonThermal Plasma (AP NTP) technology. Nonthermal plasma is a partially ionized gas generated by any potential form of power to discharge and transfer the energy to gas molecules in the surrounding. During the initiation process, the excited gas molecules then transfer the electron energy to neutral species by inelastic collisions forming highly reactive metastable free-radical species like singlet oxygen, hydroxyl, and hydroperoxyl radicals. The plasma can be generated by electrical means in the form of direct current (DC) as in DC glow discharge (e.g., Akishev et al., 1993; Machala et al., 2004), alternative current (AC) (e.g., Akishev et al., 2002; Lu and Laroussi, 2006a), or pulsed direct current (Walsh, Shi, and Kong, 2006). Ganguli and Tarey (2002) is a good reference of electricity-powered plasma sources. Radiofrequency has also been applied extensively in the past to power and to generate nonthermal plasma (e.g., Yeong et al., 1998; Guo et al., 2003)) for various applications. Recently, dielectric barrier discharge driven by pulsed electric power has also gained interest (e.g., Lu and Laroussi, 2006a \& 2006b). The thermal temperature of nonthermal plasma is low and does not have sufficient enthalpy to trigger detonation, combustion, or even to burn human skin. Thus, nonthermal plasma is commonly called "cold plasma". The reactive species generated in the nonthermal plasma react with a contaminated surface in the proximity of the gas-exiting nozzle forming a well-defined shape of glow. Figure 1 shows two photos of nonthermal plasma generated by (Figure 1a) radiofrequency power and (Figure 1b) direct-current electrical discharge. 


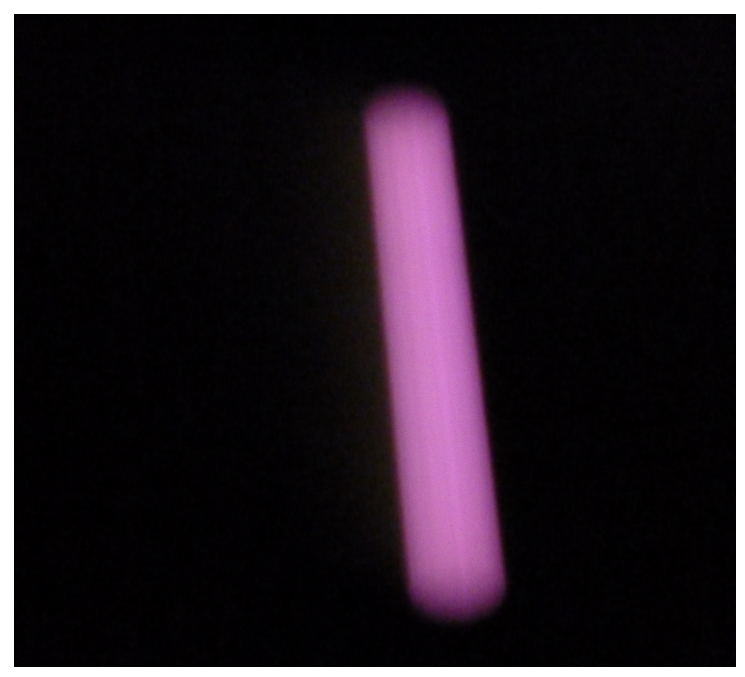

Figure 1a. Examples of AP NTP driven by radiofrequency power and shaped as a line plasma

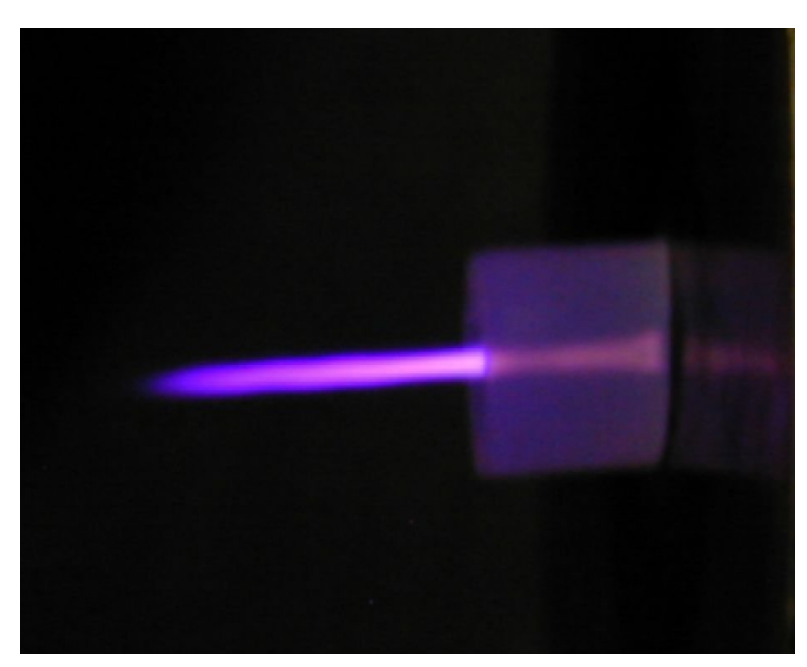

Figure 1b. Example of AP NTP driven by direct current (DC) and shaped like a round jet

AP NTP is different from a hot plasma created by laser-induced breakdown and ablation that latter was primarily used for removing heavy metals and radionuclides from contaminated surfaces in many of the nuclear complexes (Lee and Cheng, 2004a and 2004b) owned by the US Department of Energy (DOE). Previous research indicated that AP NTP is effective in the destruction of chemical and biological warfare agents (Hermann et al., 1999; Laroussi, 2002). Atmospheric pressure nonthermal plasma treatment has gained interest in the recent years in many applications such as decontamination of surfaces, medical disinfection, surface modification of polymers, and other optical devices. Laroussi (2002) and Tendero et al. (2006) provided general review of the technology.

Since AP NTP is nonthermal, it does not conduct heat to the treated surface and therefore eliminates the possibility of thermal damage. Moreover, there is no possibility for material damage by liquid since no liquid is used in the AP NTP process. Cleaning by nonthermal plasma was found to rapidly destruct biological contaminations. The times are typically in the range of seconds, as opposed to the "wet" approach that can take up to at least $30 \mathrm{~min}$. For example, destruction of B. globigii spores was reported to be within $30 \mathrm{~s}$ by Hermann et al. (1999). This high-rate cleaning can be a very important attribute of AP NTP that also does not produce liquid waste for additional treatment. There are several known advantages to using a nonthermal plasma technology for surface cleaning and decontamination, for example:

1. Ability to operate at atmospheric pressure and room temperature,

2. No residue presence after the cleaning,

3. Capable of cleaning a wide range of contaminants in a few seconds,

4. Converting waste into low toxicity or benign gaseous form (can be collected by filters or traps if needed), 
5. No liquid waste to treat or collect/store after the cleaning operation, causing no secondary pollution as a wet method would,

6. No requirement of line-of-sight like some hot plasma or photon-induced cleaning techniques,

7. High scalability enabling flexible development of a hand-held device or a scale-up cleaning system, and

8. Minimal or no damage to the treated material surfaces.

AP NTP has been applied to a wide range of problems such as removal of bioaerosols (e.g., Hermann et al., 1999) and treatment of polymer surfaces (e.g., Laroussi, 2002). Even with such a large experience base, there remain unknowns associated with applying AP NTP to intended surface decontamination technology [for military weapons systems and platforms] for removal of greases, dirt, and chembio agents. As discussed earlier, one of the unknowns is the material integrity of the surface after cleaning. Would the morphology, mechanical, thermal, optical, and electrical properties be affected after the AP NTP treatment? Another unknown is the effectiveness of the removal of contaminants to be addressed in this report, since there are no known publications in the open literature? Potential production of nanoparticles and gas species through the rapid plasma oxidation with the contaminants are contaminant dependent and the emissions remain undetermined.

\section{OBJECTIVES}

The objective of this limited-scope 12-month SERDP project is to explore the feasibility of the technology in dry removal of contaminants. The following two specific questions are addressed:

1. How effective is the AP NTP in the removal of selected contaminants without harming the support substrate?

2. What byproducts may the AP NTP technique produce? 


\section{MATERIALS AND APPROACH}

We initially proposed to investigate effects of AP NTP on metallic and non-metallic substrates during the removal of contaminants that include grease and dust. After extensive discussion with our military partners, Air Force Academy in Colorado and Eglin Air Force Base in Florida, we learned that one of the military platforms that is commonly exposed to the contaminants is aircraft frame. The material integrity of the aircraft frame is important; thus, as discussed earlier, the degradation of tensile strength after wet cleaning is unacceptable. We are persuaded to focus on using the military aircraft frame as the primary substrate in our study. This change would make the test substrate relevant to DoD applications, and also permit us to focus on addressing the objectives by not spreading ourselves too thin on two different substrates. The preparation of test samples, characterization of the samples before and after the NTP treatment, and optical assessment of the treated samples are described in this section.

\subsection{Description of Sample Substrate and Preparation of the Samples}

The substrate we used in the experiments was C-17 airframe material of 4" wide and 12 " long. The material has a honeycomb structure coated by a carbonaceous polymer layer that is embedded with metal; it is a composite material, lightweight, and structurally strong. A photograph of the airframe substrate is shown in Figure 2. Airframes are constantly exposed to contaminants. LtCol. Richard Schoske from the Air Force Academy recommended the composite material as a test substrate for this project. Since this substrate is a composite, we decided to use just this since it has both metallic and nonmetallic components in its structure. This way, we could focus on achieving the objective more effectively within our limited resources. All the experiments were done on the composite airframe material, except those on glass slides for some additional tests.

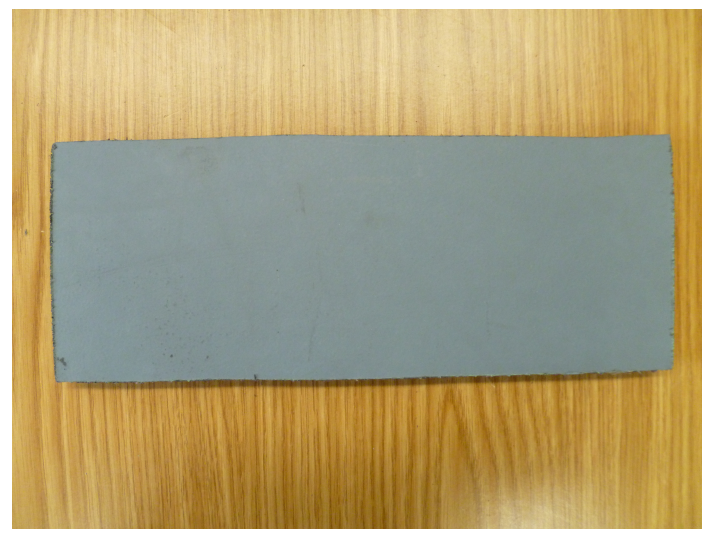

Figure 2a. Photo showing an airframe sample used as the substrate in this project.

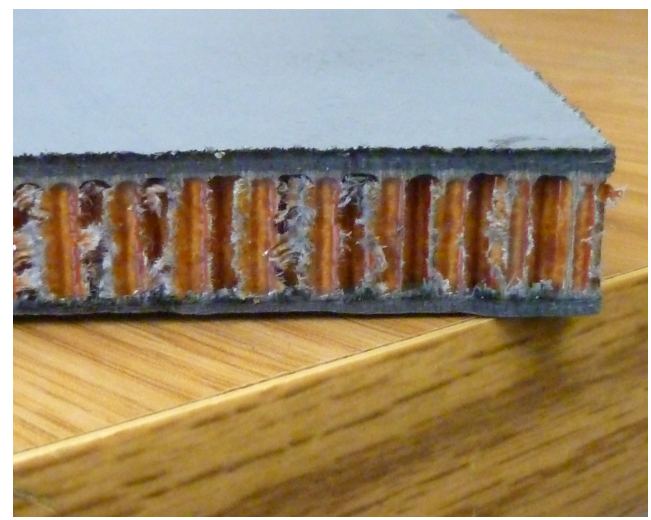

Figure $2 \mathrm{~b}$. Photo showing the cross section of an airframe sample used as the substrate in this project. The honeycomb structure is sandwiched in between two layers of metallic-polymer skins in light blue color. 
The contaminants that were used for testing AP NTP removal include (1) marker stain, (2) oil grease, (3) a chemical warfare simulant, and (4) pollen grains. The marker stain was a blue color Hi-Liter fluorescence marker made by Avery Dennison in Framingham, MA. The oil grease was vacuum pump oil from Liqua-Tek, Inc. at Oconomowoc, WI. The chemical warfare simulant Triisopropyl phosphate (TIP), as the simulant for VX, was obtained from Sigma Aldrich. TIP is a stable compound under normal room temperature and pressure, but is a mutagen upon exposure above the permissible level. TIP is moisture sensitive, and it will decompose into phosphine, carbon monoxide, oxides of phosphorus, and carbon dioxide. The pollen grains were of Ambrosia species (commonly called as ragweed) from the Greer Lab in Lenoir City, NC. Pollen grains are very hardy materials, primarily composed of carbon. The elevation of pollen counts has strong seasonality. Contamination by pollen grains varies with season and locality. A pollen grain is the vehicle of plant reproduction and can travel in the air over a great distance. The first contaminant (i.e., marker stain) listed above was never used on the airframe substrate for testing, but was used on a glass slide to allow for use in optical microscopy to visualize the removal process.

Although we spread TIP and oil grease to form thin layers of complex organic composition, these species do not form a homogeneous thin layer on a substrate surface in a real application. A thin layer model is only for our experimental convenience and to establish a condition where we can duplicate work. In the real world, these liquid droplets deform upon impaction and deposition on a substrate surface resulting in some spread, while a pollen grain does not deform nor spread into a smear. Pollen is a bioaerosol particle. Once pollens deposit on a surface, the grains stick on the surface as a single entity without any spread. The rigid pollen structure serves as a protective barrier of the antigens that are typical of organic molecules that could be destroyed by AP NTP. Therefore, we anticipate that the physical structure of the pollen contaminant to would produce a significant contrast to the thin organic layer in the AP NTP removal mechanisms.

\subsection{Description of laboratory-scaled nonthermal plasma sources}

A bench-scale nonthermal plasma source was assembled during the first couple of months of this project. This first plasma source was based on either direct current or pulsed direct current to initiate gas breakdown leading to the formation of nonthermal plasma. The plasma configuration from this power source yielded a "jet-like plasma" (see Figure 1b). The diameter and length of the plasma jet can be varied by controlling voltage, flow rate and pulse manipulation (pulse length and height). In other words, we could stretch plasma from a few $\mathrm{cm}$ to $30 \mathrm{~cm}$ in length by varying the above-mentioned parameters. The setup of this plasma source was to enable us to identify a possible working condition where air and or water vapor can be used to generate nonthermal plasma, because all current AP NTP technology operates on a gas mixture of helium and other gases. Helium was the main gas to initiate the plasma, but helium is expensive. Furthermore, large amount of helium is generally needed making the helium-based AP NTP not cost effective or attractive to the decontamination sector. 
We attempted a second plasma source as initially proposed. This second source was based on the design of Lin and Cheng (2002), Lee and Cheng (2004a and 2004b), and Cheng et al. (2006) using a laser-induced breakdown instead of pulsed electrical breakdown to generate transition plasma. Note that laser-induced plasma contains higher number $\left(\sim 10^{19}\right)$ of electrons and ions than that of electrical plasma by one to two orders of magnitude; thus, theoretically, we could provide a more effective plasma source than the electrical power. After a couple of months of trials using various gas mixtures and combination of laser power parameters (pulse width, energy, and repetition rate), we were unable to produce and maintain continuous uninterrupted nonthermal plasma based on the laser-induced breakdown approach. We hypothesized that was because the repetition rate of the pulse laser firing was too slow (variable with a maximum of $20 \mathrm{~Hz}$ ) to maintain the free radicals population. Our laser firing repetition rate was 3-4 orders of magnitude slower than the rate we used to achieve nonthermal plasma production by electrical power.

Finally, we acquired a commercial nonthermal plasma source for additional tests. This plasma source was operated on helium/oxygen gas mixture and was driven by radiofrequency (RF) power. The coupling of RF power at $27 \mathrm{MHz}$ to the gas mixture creates a condition where breakdown occurs and can be sustained for production of AP NTP. It is important to note that the max power of this unit is $200 \mathrm{~W}$, which might not be sufficient for removal or destruction of particulate material. This commercial instrument is described in the Section below.

\section{Powered AP NTP Source}

Figure 3 shows the internally built NTP source that is powered by DC in CW or pulsed mode typically at several thousand volts. In the pulsed mode, the pulse rate is up to

$2 \mathrm{k} \mathrm{Hz}$. The black circular plate at the top of the vertical $\mathrm{T}$ serves as the anode, while the needle inside the plastic cover delivers the discharge of plasma that consists of a mixture of ions. An example of the discharge of NTP powered by continuous DC is shown in Figure $1 \mathrm{~b}$ as a round jet with a length of nearly $25 \mathrm{~cm}$ and diameter approximates the exit nozzle about 2-5 $\mathrm{mm}$. This setup was used to investigate the removal of marker stain from a glass slide, representing substrate such as a computer monitor or LCD screen. The gas mixture used in the experiments is a mixture composing of helium, water vapor, and air. Helium is a research grade gas of $99.9 \%$ purity, while water vapor is maintained at the room temperature at $21-22^{\circ} \mathrm{C}$. Thus, we anticipate that the radicals to be produced by this setup

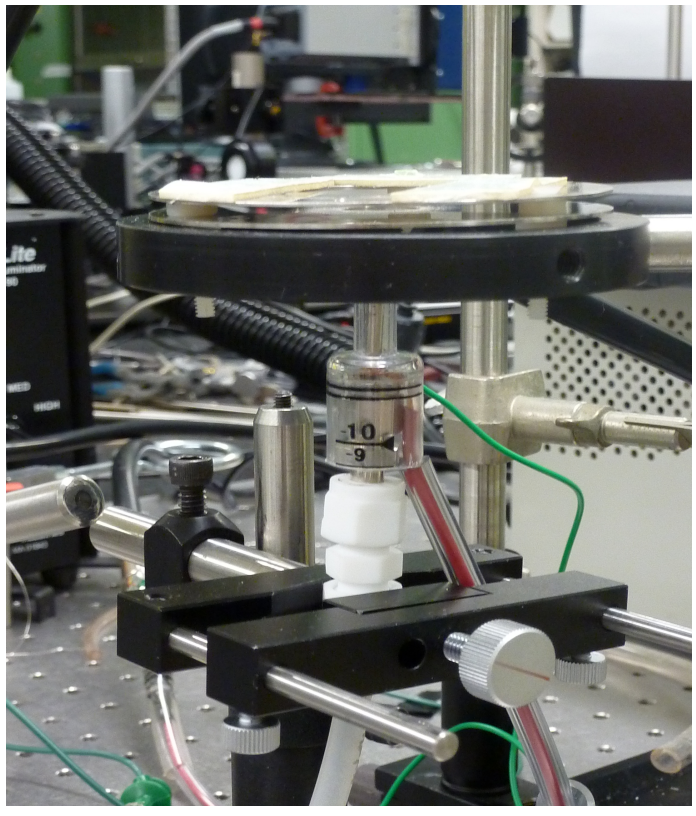

Figure 3. Photo of Experimental Setup of DC-NTP 
consist of $\mathrm{O} \bullet, \mathrm{OH} \bullet$, and $\mathrm{O}_{3}$. In fact, we could smell the presence of ozone at the start of the NTP forming due to the corona discharging.

\section{Radiofrequency Powered AP NTP Source}

A commercial AP NTP source operated on RF power was tested, also, during this project. The commercial instrument is shown in Figure 4a. The box with black panel at the back in Figure $4 \mathrm{a}$ is the control and driver of the plasma source. The frequency of the RF the instrument operated is $27.12 \mathrm{MHz}$, and the power operated was at 200W. The unit was designed to operate from 0 to $300 \mathrm{~W}$. The application head where the plasma was emitted is shown in Figure $4 \mathrm{~b}$ and the plasma is shaped like a glowing sheet (as shown in Figure 1a). The exit nozzle in Figure $4 \mathrm{~b}$ is linear about 2" $(5.1 \mathrm{~cm})$ in length and weighs about $0.25 \mathrm{lbs}$

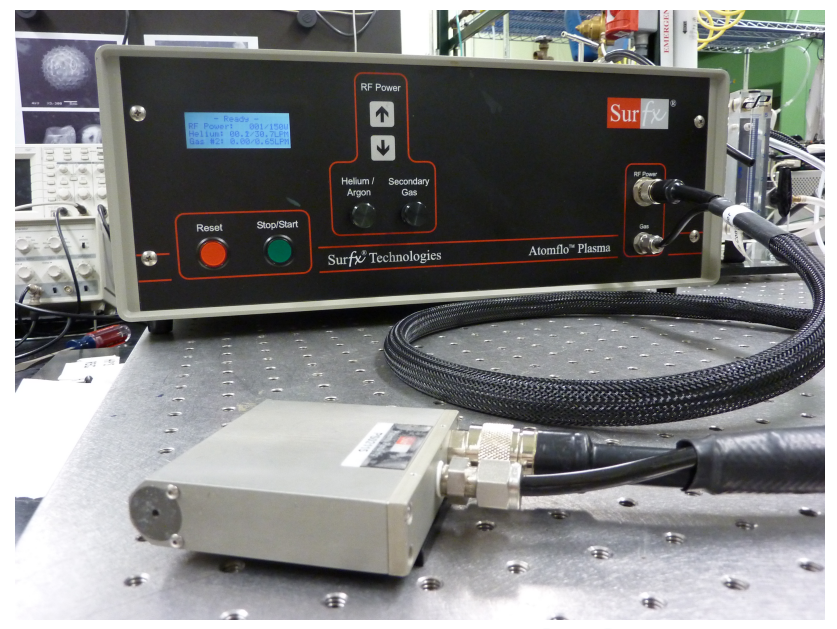

Figure 4a. AP NTP from Surfx Technology

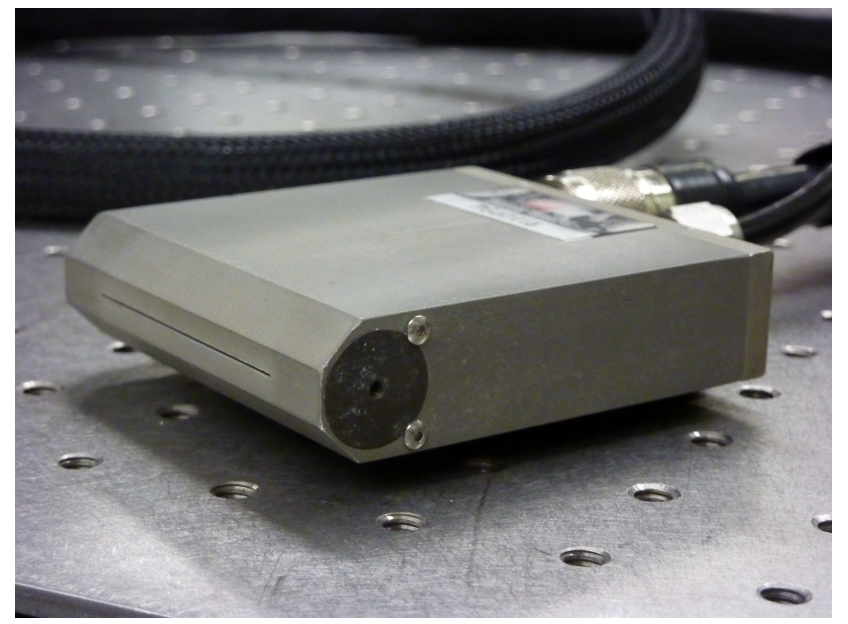

Figure 4b. Close up of the AP NTP Head

(114g). The operating gases used in this instrument are helium and oxygen with a mixture composition of $(95 \%$ at $30 \mathrm{lpm} / 5 \%$ at $1.5 \mathrm{lpm})$. The source-to-sample distance was operated at about $10 \mathrm{~mm}$ (the manufacturer recommended range is 3 to $15 \mathrm{~mm}$ ). With helium and $\mathrm{O}_{2}$, the operating gases of this unit, we anticipate that the radicals produced by this unit are mostly $\mathrm{O}$ and $\mathrm{O}_{3}$. Since ozone is also present, we conducted this experiment inside a ventilation hood enclosure. Figure 4c shows an example of the plasma source on

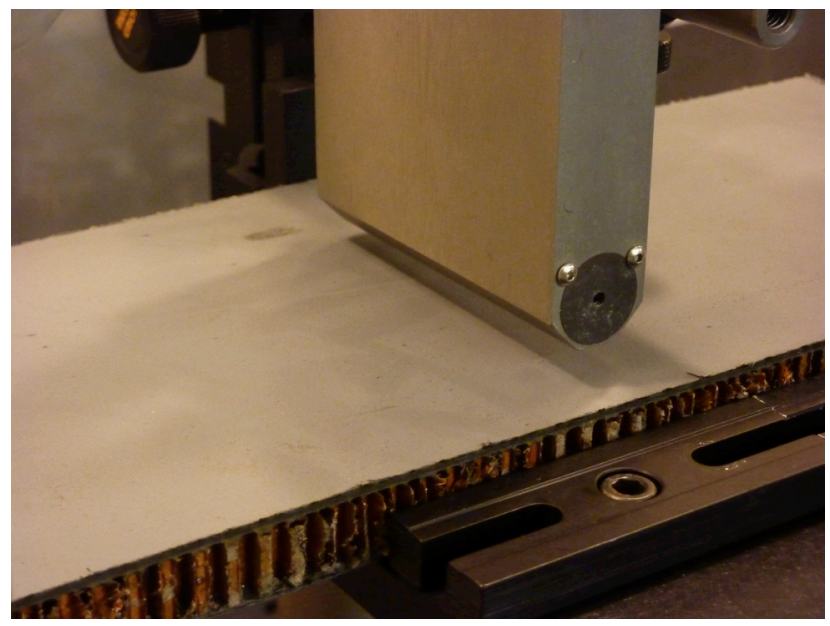

Figure 4c. Display of RF powered AP NTP on a airframe sample. 
the airframe substrate during a treatment. The head is positioned close to the substrate surface to facilitate the reactions by free radicals. The experimental setup is in a ventilation hood for environmental safety and health precaution.

\subsection{Characterization of the material surfaces}

Characterization of the original/control surfaces and the treated surfaces yields information to understand the cleaning performance. For this proof-of-principle research project, we proposed a set of limited characterization measures such as visual inspection to be reduced by using a digital camera and image analysis. More extensive characterization is required to ensure the material integrity after a NTP removal, but surface texture is the first order approximation of the integrity without invoking a detailed material analysis.

The proposed set of characterization consists of the following:

1. Optical microscopy examination of the substrate surface before and after the NTP treatment.

2. Scanning transmission electron microscopy of selected substrate samples.

3. In-line nanoparticle characterization by a commercial instrument, the Scanning Mobility Particle Spectrometer, to monitor production of ions and nanoparticles.

4. In-line charge characterization by an aerosol electrometer.

5. In-line spectral analysis of the plasma and optical emissions to identify species that are produced by the treatment.

\section{RESULTS AND DISCUSSION}

We have investigated a number of variables that were considered important to the deployment of this technique at a larger scale. The composition of the carrier gas is one of the variables because the composition affects the final population of reactive species as well as the operational cost.

\subsection{Effects of Composition of Carrier Gas Mixture on Removal}

The effect of gas composition on the AP NTP plasma cleaning can be seen in Figure 5. This AP NTP was powered by a direct current technique $(6 \mathrm{kV})$. We purposely operated this NTP at low power to test the reaction at an extreme operating condition. The total volumetric flow rates of these gas mixtures were kept at 2 slpm in all experiments. The curves displayed show the removal rate of $25 \mu \mathrm{g}$ color stains (marker or methylene blue of $1 \mathrm{~cm}$ diameter circles) on glass slides. The optical transmittance (OT) measures the amount of light passing through the stain. After the plasma removal operated for a given length of time (given in the X-axis), the OT value is expected to increase due to degradation of the coated material on the glass substrate. Since NTP has little enthalpy and does not "burn" the material as in the combustion sense, we did not anticipate NTP to turn organic molecules into black carbon through pyrolysis. Therefore, the slope of a curve indicates the 
removal rate. The OT value for a clean slide is constant and close to 1.0 as shown in the light blue color with a legend "CleanSlide". Most curves are upward with the exception of the $\mathrm{HeO}_{2}$ mixture, which showed a downward trend. Duplicated experiments with $\mathrm{HeO}_{2}$ as the carrier gas did not consistently yield the downward trend; some were even reversing the downward trend. We did not know what might have caused the downward trend for the case using $\mathrm{HeO}_{2}$. We left this case in Figure 5 hoping that it might remind us that this case needs to be looked at. Note that using helium case in a field operation may be costly so the case of $\mathrm{HeO}_{2}$ may just be a moot point.

The comparison of the curves shows that the removal generally increased from OT of 0.6 to 0.8 by the mixtures of (1) $\mathrm{AirH}_{2} \mathrm{O}$ and (2) $\mathrm{ArO}_{2}$. Other combinations appear to level

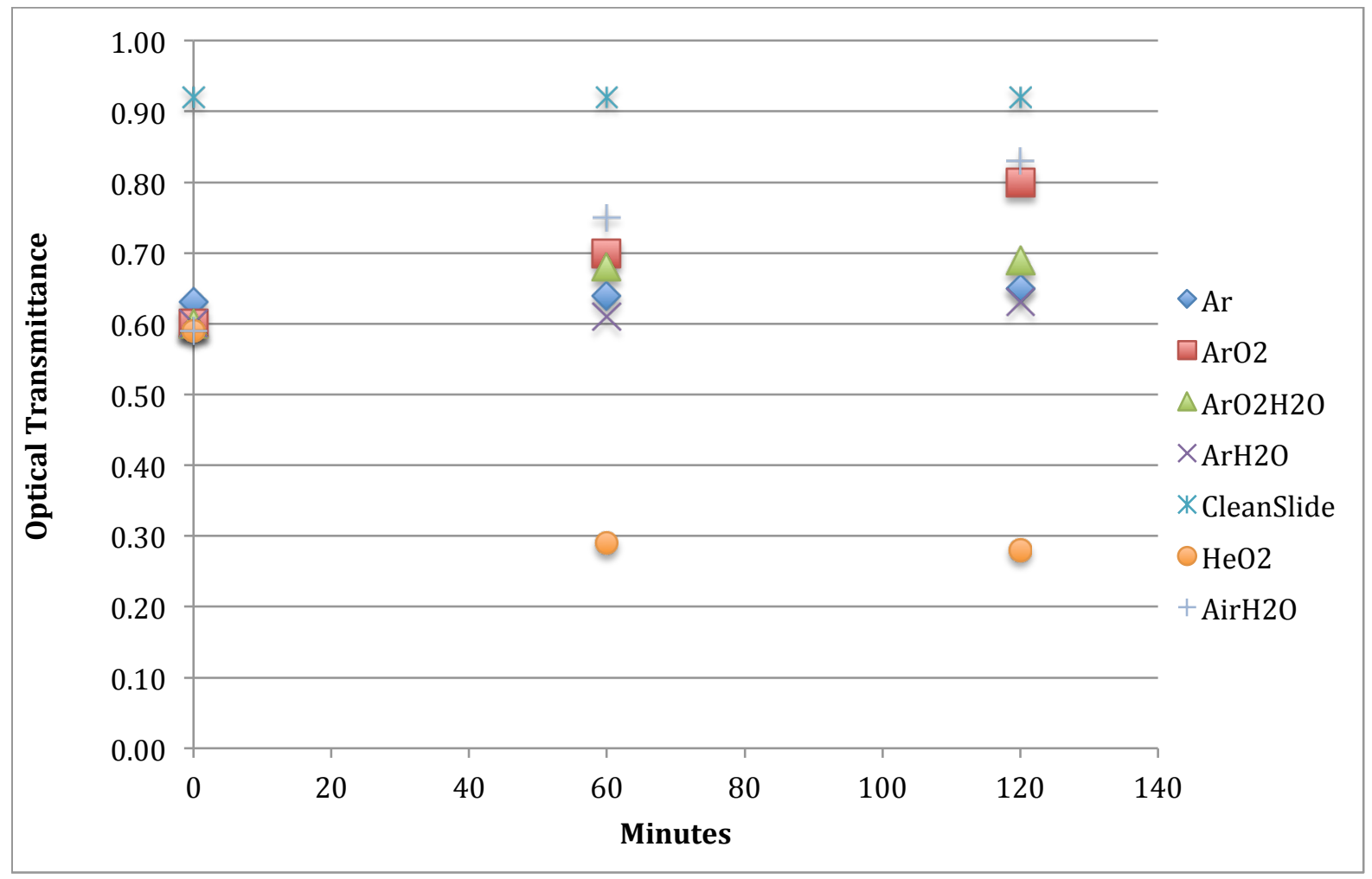

Figure 5. Plot of optical transmission verse time of exposure in minutes

off at less than 0.7 after an hour of treatment. This provides an encouraging result showing that a scale-up NTP may be feasible for field operation on large weapons systems and platforms by using a mixture of air (particle free) and water vapor (at $21^{\circ} \mathrm{C}$ ) instead of expensive gases like $\mathrm{He}$ and $\mathrm{Ar}$ in a large-scale field operation.

\subsection{Evaluation of Surface Damage by Heat Associated with the Plasma}

Damage to the substrate material may cause mis-interpretation of the results, since the damage could bias the performance assessment of the NTP removal. The reactive gas emitted from the nozzle for decontamination use does not have sufficient enthalpy to heat, 
but the radiofrequency power could heat the nozzle head of this commercial unit. Since the distance between the substrate surface and the nozzle head is short $(\leq 10 \mathrm{~mm})$, the hot nozzle body could impose thermal removal process on the surface by transferring radiant heat to the surface. Further, the heat could be added to the gas flowing through the nozzle thereby making the reactive flow hot. This would lead to additional removal that unfortunately could contribute to biased experimental data that could have been prevented. To mitigate the thermal effect, we determined the optimal distance for the NTP removal experiments, factoring in the concern of thermal damage and effective removal by reactive gases, by conducting damage assessment first.

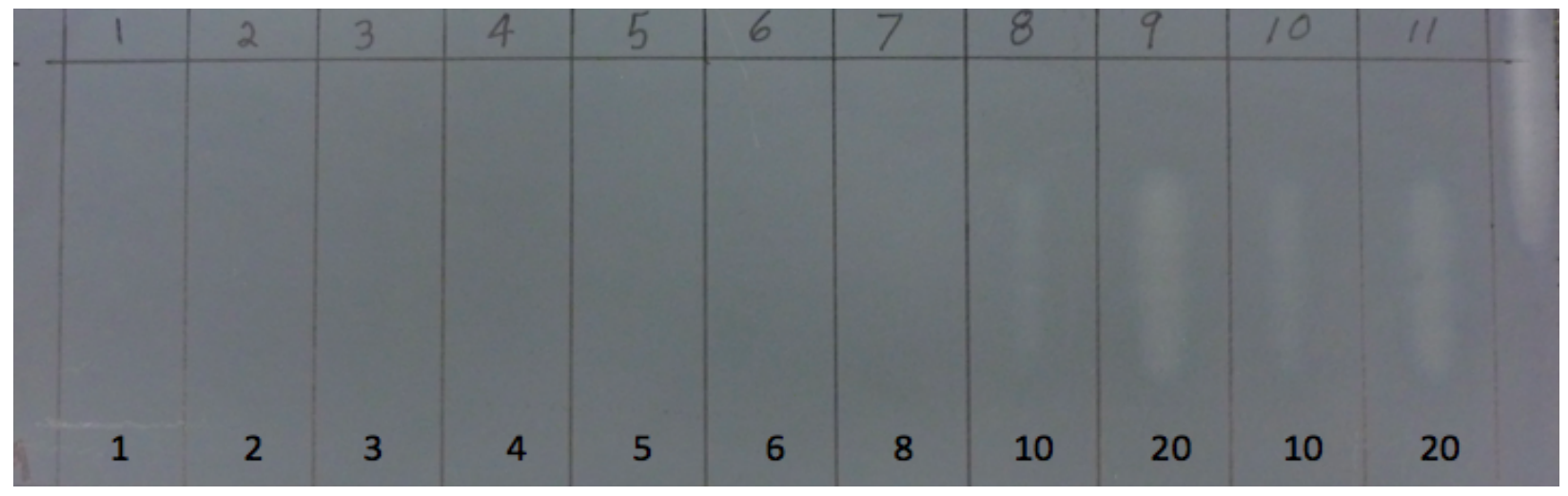

Figure 6. A photo showing substrate under damage test

We used a K-type thermocouple probe to measure the gas temperature as a function of distance from the source nozzle tip at the head. Operated at $200 \mathrm{~W}$ power, the temperature was about $160^{\circ} \mathrm{C}$ at the source, and $110^{\circ} \mathrm{C}$ at about $15 \mathrm{~mm}$ distance from the source (a maximum application distance suggested by the manufacturer in the manual). The numbers in Figure 6 indicated at the bottom show the duration in minutes that the NTP was on. The distance between the head and sample substrate surface was set at a distance of $10 \mathrm{~mm}$. As shown in Figure 6, there was no discernible discoloration up to 8 minutes. In other words, it appears that the airframe substrate was able to resist the heat at $110^{\circ} \mathrm{C}$ for about 8 minutes before the surface property was modified and started to appear reflective. Also reactive gas molecules might have contributed to the visual discoloration, but the data suggested the operating time should be less than 8 minutes when using this commercial head to be safe in preventing thermal damage to the surface of the substrate.

The vendor indicated that the instrument head could be retrofitted with a cooling unit such that the gas from the plasma would be cool to touch at $10 \mathrm{~mm}$ or shorter. Our experience also suggested that if future development of the instrument is warranted to operate at a power higher than operated for this project, a cooling unit (e.g., circulating water through the head) would be required.

Although there are many American standard material testing methods available, we did not use any of them to evaluate details of the material properties; e.g., mechanical or 
structural properties of this airframe due to resource limitation. We felt detailed testing should be done for more specific application. During the exploration phase of this limitedscope project, the visual discoloration was a clear evidence of potential surface damage.

\subsection{Removal of Oil Grease and CWA coated surfaces}

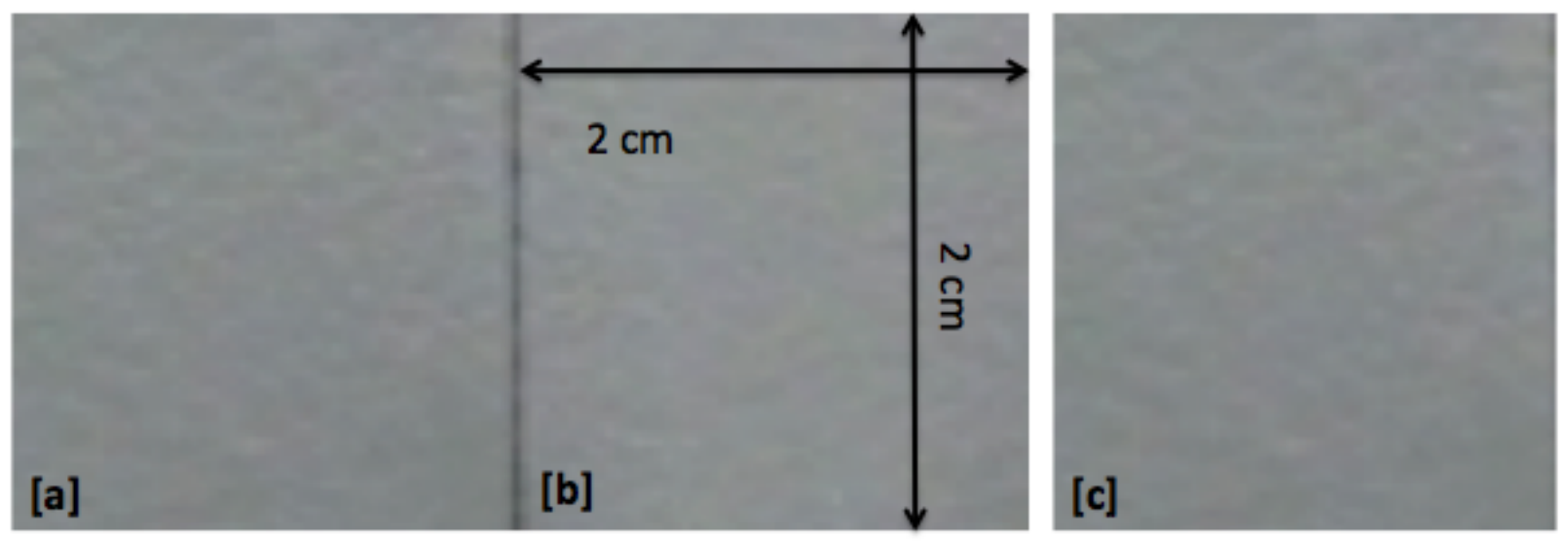

Figure 7. Removal of Oil Grease. [a] Untreated original substrate surface, [b] Oil treated surface of the same area as shown in [a], [c] RF-NTP treated surface of the same area as shown in [a] and [b]

Oil and grease are common contaminants in many weapons platforms and systems. This category of contaminants consists mostly of organic molecules with a minute amount of metals in it. An example of the removal of an oil film attached to an airframe substrate by the RF-NTP is displayed in the 3-panel Figure 7. The left panel [a] shows the untreated original airframe surface, [b] the oil-treated area same as the one in the left panel, and [c] the same area as in [a] and [b] that was oil-removed by RF-NTP. The oil was spread to form a film covering a round area that was inscribed by an approximately $2 \mathrm{~cm}$ by $2 \mathrm{~cm}$ square. We used this square area and assume that all the oil was in it and our estimate for the thickness was about 25 microns.

Comparing the two panels [b] and [c] pixel-by-pixel in image analysis, we concluded that the oil was completely removed, leaving two pixels of the same spot of identical value. It appears that the oil did not chemically react with the airframe surface to alter its pixel value even after complete RF-NTP removal. Comparing pixel-by-pixel of images in [a] and [c], we found that the RF-NTP did not alter the airframe surface, because of the same reason of identical pixel values. Thus, we concluded that the surface of the substrate was virtually not changed and the oil contaminant in a thin layer of 25 microns thick was completely removed by the RF-NTP technique. The thicker the layer the longer the NTP dwelling time to reach complete removal.

We also applied spectroscopic and aerosol detection techniques during the RF-NTP treatment of the oil-contaminated airframe substrate. The laboratory setup is shown in Figure 8. One Scanning Mobility Particle Sizer (upper left; SMPS TSI Model 3069L) was 
used to measure particle size distribution of any possible nanoparticles that might be produced by the removal process. An electrometer (lower left, TSI Model 3068B) was used to measure the particle-bound charges produced during the removal process. One spectrometer was set up at 90-degree viewing angle (SP2) from the plasma sheet, while the other was along the long-direction of the sheet (SP1). The difference between these two directions was that SP1 could receive a higher signal than that observed at SP2. Plasma is in the direction normal going to the paper at the purple line position. The head is about $10 \mathrm{~mm}$ from the surface. SMPS is the TSI Scanning Mobility Particle Sizer Model 3089L equipped with a long DMA and UCPC (Model 3025A), aerosol electrometer is TSI model 3068B. SP1 is an OceanOptics spectrometer HD2000, while SP2 is a high resolution Acton spectrograph equipped with an Andor ICCD.

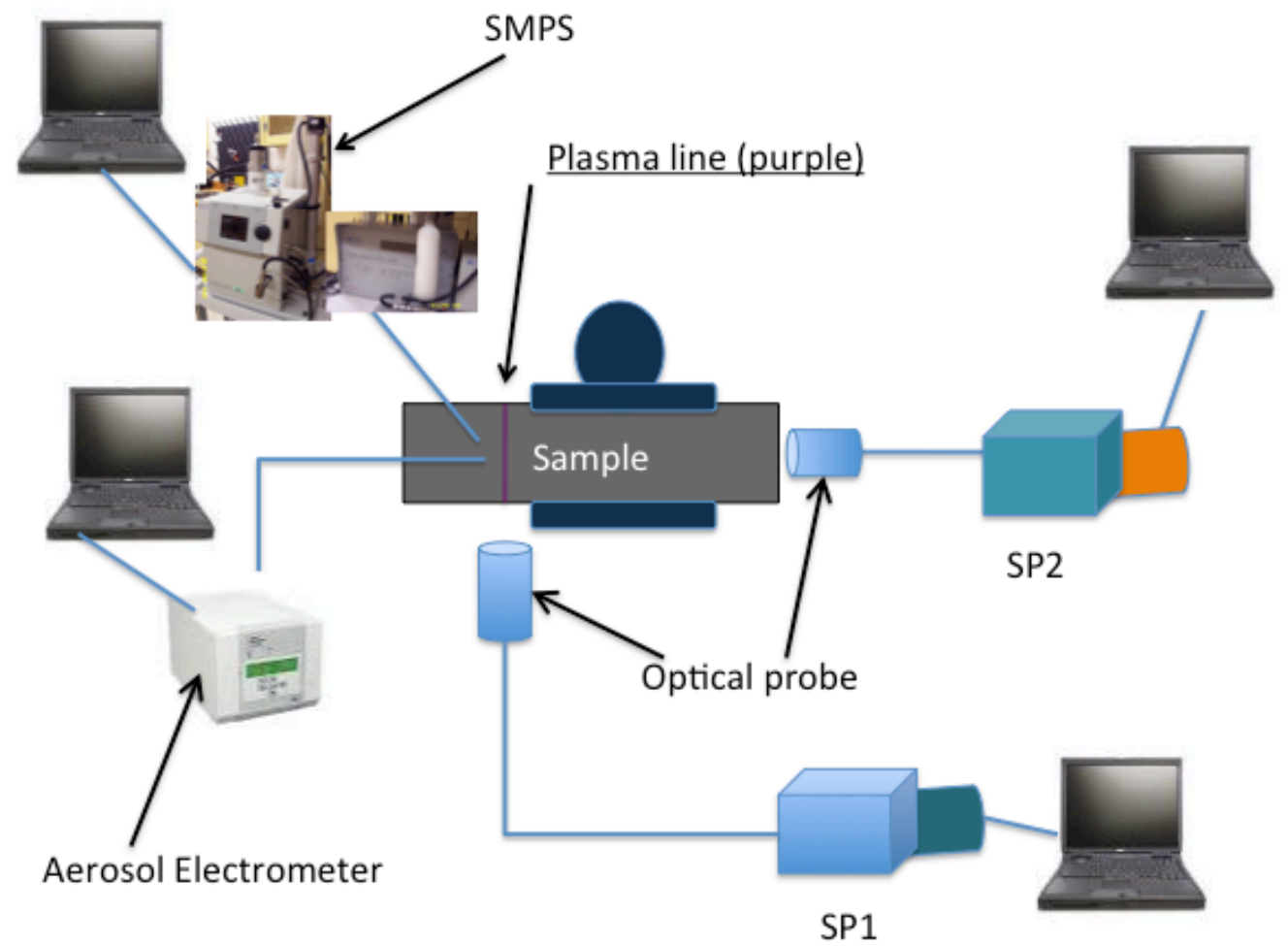

Each instrument is controlled by individual computer.

Figure 8. Setup for spectroscopic and aerosol detection.

If RF-NTP removal of oil molecules from surface of the airframe produced charged particles, they could be detected by a Faraday Cage used by the Aerosol Electrometer 3068B online in near real time. We did not use any charge neutralizer as did in SMPS. This way we could not calculate the particle concentration because we do not know the number of charges for each particle. However, we were only concerned with the total concentration of charges measurable by 3068B. The particle data measured by SMPS are presented in the following section. 
Aerosol electrometer (3068B) registered a 600 to 5,000 folds increase in charge concentration over a background level during the time when the RF-NTP was on the substrate. The background particle count in the experimental chamber is typically less than 400-500 particles per cubic centimeter and all of them were less than $30 \mathrm{~nm}$. This result indicates that the oil was removed by the plasma and oil molecules were possibly fragmented into ions during the removal process. Since the NTP did not stay on long enough to exceed the thermal damaging time as discussed previously, it is unlikely these charges would have been produced by the thermal effect. There were no charged particles produced and detected by the $3068 \mathrm{~B}$ during a side test using a heat gun.

Whether or not there was a significant quantity of charged ions produced as shown in the example above, the chance of particle (in the size range of $50 \mathrm{~nm}$ or less) formation during the plasma treatment is high. A common technique to detect the presence of nanometer scale particles such as these is to use SMPS as mentioned above. The size distributions of the nanoparticles observed by using the SMPS are shown in Figure 9. These distributions were the lognormal fits to the measurement data. The peaks of these distributions are centered at about $7 \mathrm{~nm}$ indicating they could be formed as a result of heterogeneous condensation due to the increase of molecular and ion concentrations in the cold region of the plume. Different colors represent different oil layers of thickness (or different volumes of oil) added to the same area, which ranged from 100 to $350 \mu \mathrm{L}$. The total mass of each curve integrated from a size distribution is on the order of pg per cubic meter. This mass concentration is currently impossible to detect with any existing aerosol instruments available for in-situ real-time particle mass detection.

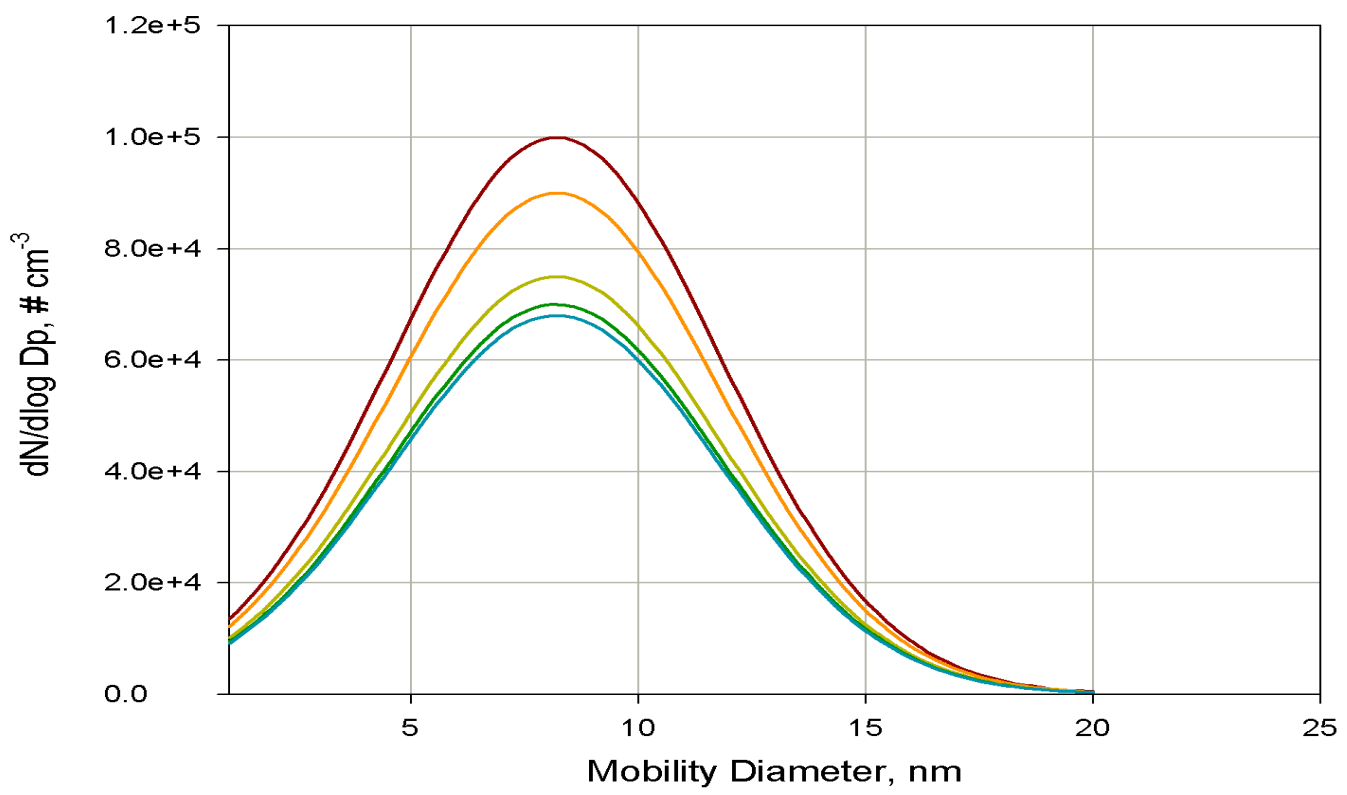

Figure 9. Size distribution of particles from RF-NTP treatment on oil layered airframe substrate 
It is worthy to note that similar (single mode) size distributions when airframe substrates were coated with a thin TIP layer. The peak location of the particle size distribution is also located in the 6-10 $\mathrm{nm}$ region, but the peak height is an order of magnitude higher than those in Figure 9. When more CWA is added, we also observed the increase in peak height but the peak location remained unchanged. We think TIP molecules might be broken down by NTP more thoroughly than oil leading to the higher peaks. We did not perform detection using the mass spectrometer available to us, because we doubt if it is possible to detect the species at such a low quantity $\left(\mathrm{pg} / \mathrm{m}^{3}\right)$. However, we were confident that the data suggest the nanoparticles were indeed produced by the decomposition of the coated materials.

Figure 10 shows an example of the optical emission spectrum observed from SP1 when NTP was on CWA-coated airframe substrate. This spectrum was taken with an Echelle spectrometer (SE-200) with a 30-millisecond exposure time. The prominent peaks identified are located at 706 and 777, while there are several small peaks scattered between 300 and $700 \mathrm{~nm}$. The insert shows a mercury light source spectrum with the major peak at $253.7 \mathrm{~nm}$ in the UV region. The 706-nm line is from the helium atom, while the 777$\mathrm{nm}$ line is for oxygen radical. We did not attempt to identify the smaller peaks, as they are mostly likely molecular fragments decomposed by the NTP during the treatment.

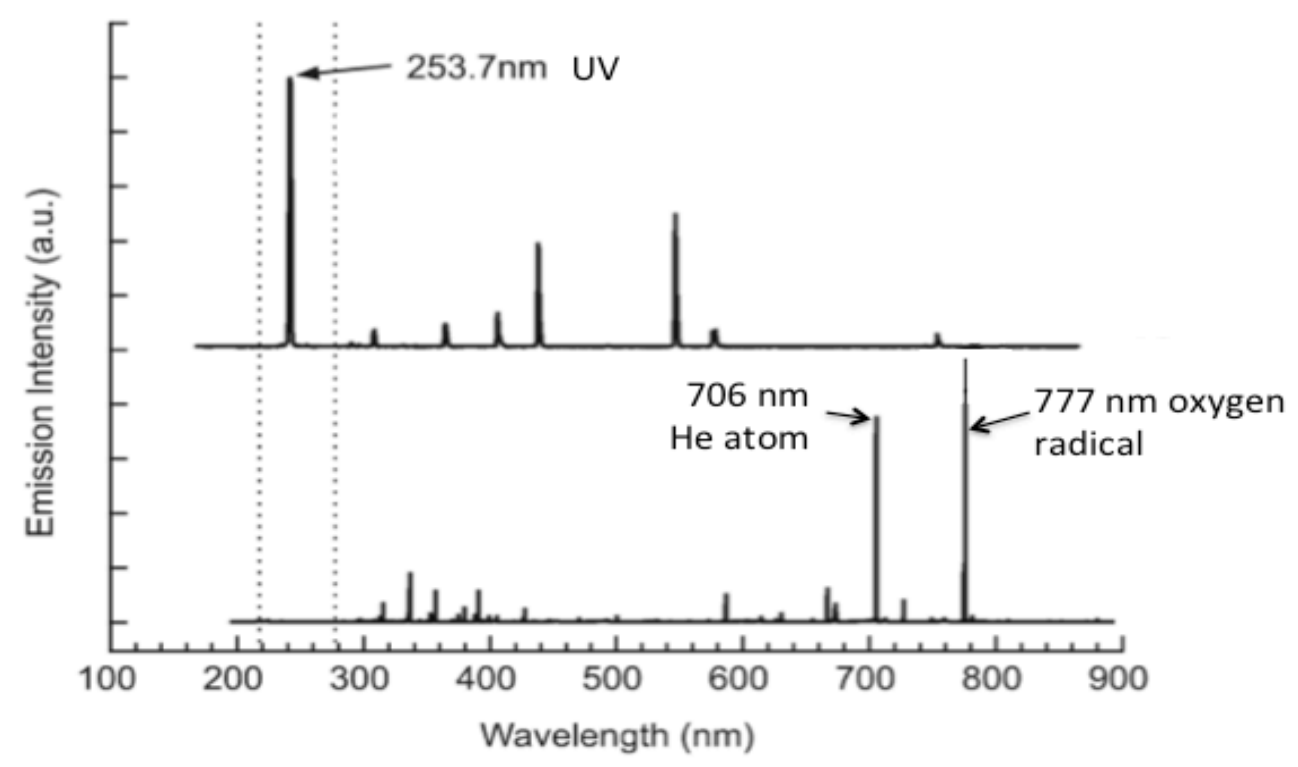

Figure 10. Spectrum of RF-NTP on CWA coated airframe substrate 


\subsection{Removal of Pollen Grains}

During this research, we considered using plasma for the removal or disintegration of particles without damaging the substrate surface underneath the particles. This is a challenging problem because applying laser higher energy to vaporize the deposited particles on a substrate surface without impacting the surface as in Lin and Cheng (2002) through the laser energy or the heat generated by focusing the energy is known to be a difficult task. NTP in theory enables us to revisit the problem and opens the door for a new opportunity for such an application. Environmental particles can be inorganic as in dusts and biological as in pollen grains. Both types of particles are commonly encountered in the indoor and ambient environments. We selected Ambrosia pollen grains for this study as pollen grains were in our original proposal as a test contaminant. Ambrosia is common pollen and many people are allergic to it. The left panel of Figure 11 shows a scanning electron microscopy photo of an Ambrosia pollen grain, while the right panel shows the distribution of the yellow pollen grains spread on a glass slide.

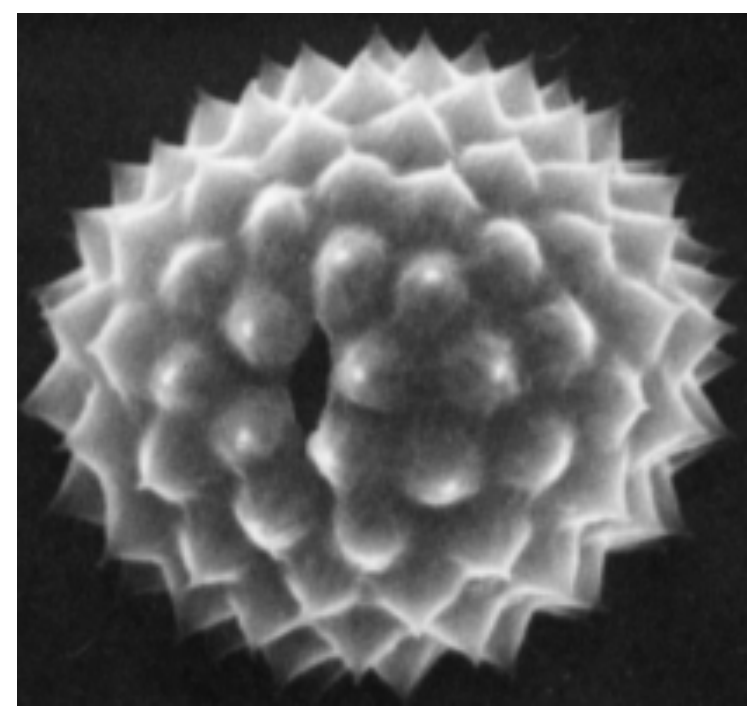

Figure 11a. Scanning Electron Microscopic Image of An Ambrosia Pollen Grain

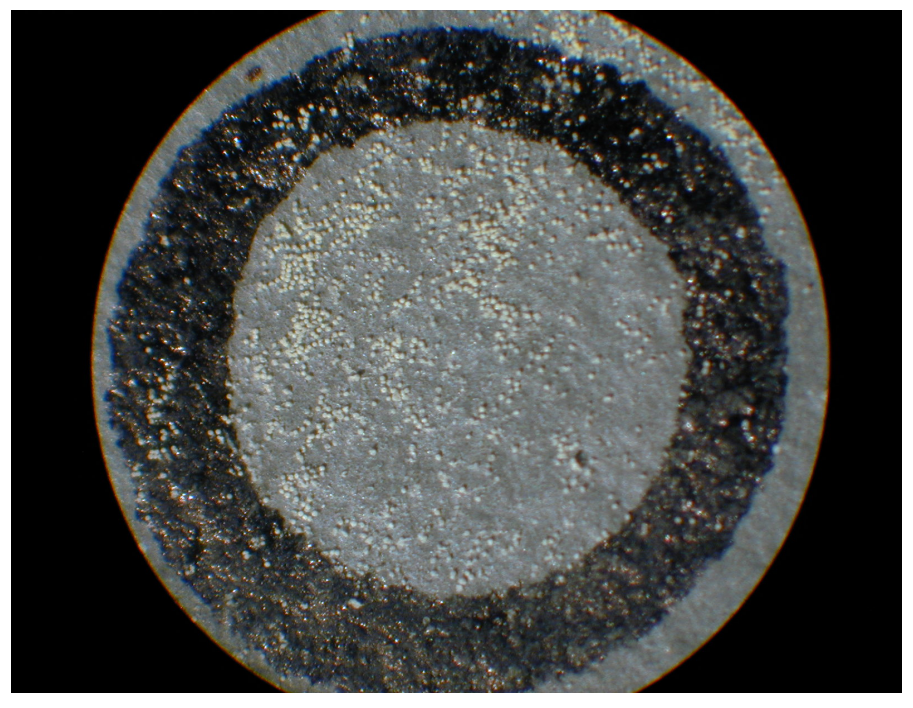

Figure 11b. Image of Ambrosia Pollen Grains deposited on a glass slide

We applied Raman spectroscopy to the Ambrosia pollen grains before and after NTP treatment. Figure 12 shows four spectra taken before treatment ( $0 \mathrm{~min}$ ) and for 3, 5, and 10-min of treatment times. Three main Raman bands were identified for the pollen grains before NTP was applied. Two major bands were at 1518.7 and $1594.9 \mathrm{~cm}^{-1}$ that correspond to carotenoid $v 1$ (the first vibrational mode) and protein phenylalanine or tryptophan, respectively. The $1152.5 \mathrm{~cm}^{-1}$ is likely to be carotenoid $v 3 \mathrm{C}-\mathrm{H}$ or $\mathrm{C}-\mathrm{C}$ stretching. These identified bands are consistent with the literature (Schulte et al., 2008). As shown in Figure 12 , the intensity of the Raman signature was decreased as the treatment time increased, indicating the amount of allergen was decreased. The removal rate was high from 0 to 3 min, and slowed down from 3 to $5 \mathrm{~min}$. The removal leveled out after five minutes, possibly indicating the allergen was completed removed. Not only the Raman spectra after 5 min of 
treatment leveled out but also the vibrational band structure of the allergen molecules was no longer as visible as that of the untreated sample at 0 and $3 \mathrm{~min}$. The disappearance of the Raman structure indicates that the allergen molecules were removed from the surface or possibly decomposed.

There are biochemical assays that can be applied to test the potency or reduction of the pollens after NTP treatment. For example, one can assay for IgE that is an antigen responsible for pollen allergenic reactions. We did not pursue this further because it is not within the scope of this project. From our previous experience in treating organic molecules, we hypothesize that an antigen could be destroyed during the treatment even if the pollen grains appear intact in its appearance. AP-NTP is not a line-of-sight treatment technique; it is possible that some reactive species from the plasma could enter the confined space of the pollen structure and react with the antigens denaturing these allergens to be non-allergic even though the outside pollen cage looks intact.

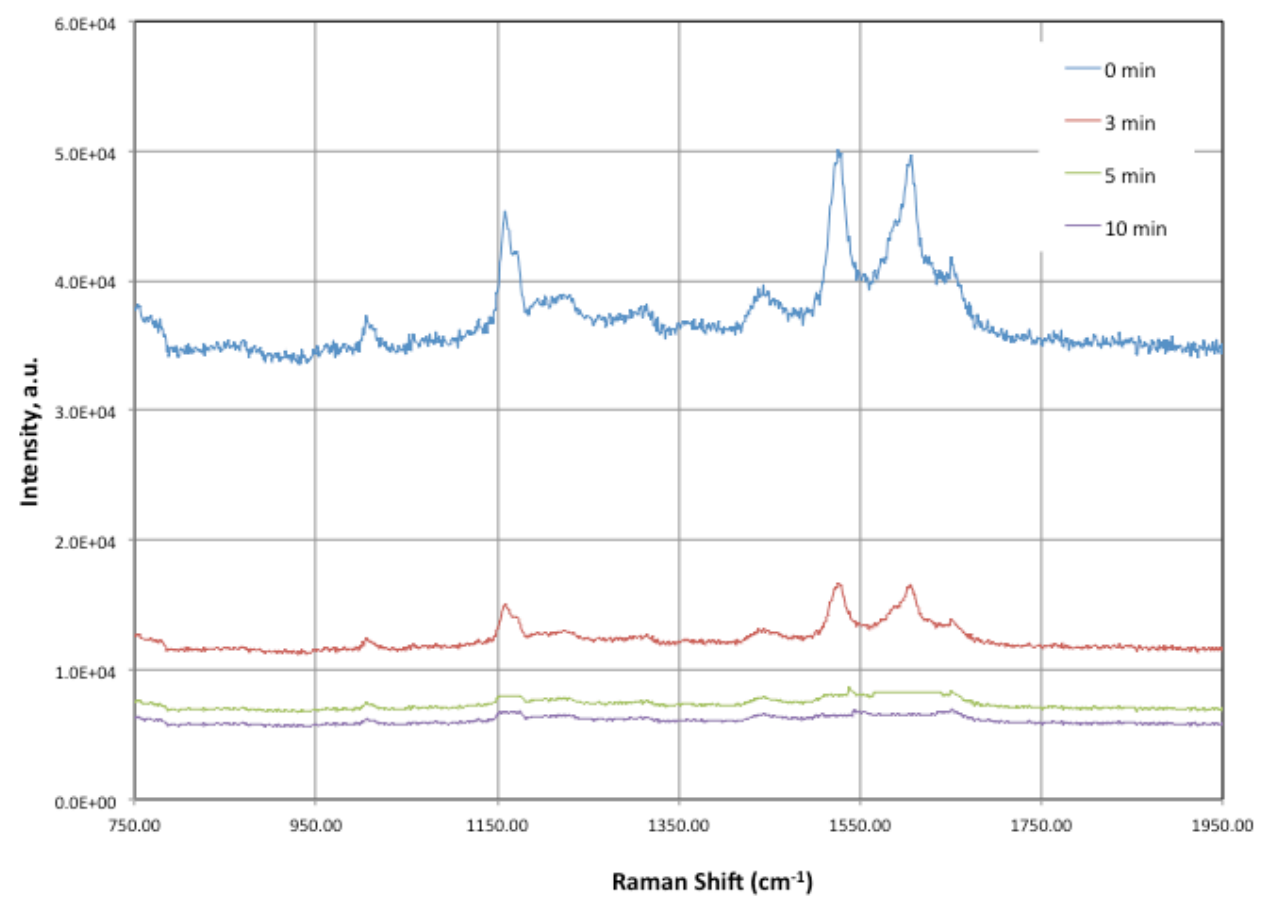

Figure 12. Raman Spectra of the Untreated Ambrosia Pollen Grains (0 min) and Treated after 3, 4, and $10 \mathrm{~min}$. 


\section{CONCLUSIONS AND RECOMMENDATIONS}

The conclusions from this limited-scope project study are:

(1) Atmospheric nonthermal plasma can be an effective source for the removal of surface contamination on weapons systems and platforms.

(2) The removal approach does not use any liquid therefore producing no secondary wastewater to be treated.

(3) The nanoparticles produced are detectable but can be safely controlled and removed by a HEPA filter.

The recommendations are:

(1) A cooling module is required to remove the heat generated by the power source.

(2) The approach of using a mixture combination of air and water as a carrier gas with a minimal plasma triggering gas feed should be pursued as this will be a significant cost saving in the scale-up application.

(3) Development of a scale-up AP NTP is required for moving the treatment technology for testing on fielded weapons systems and platforms. This can be achieved through Environmental Security Technology Certification Program.

\section{ACKNOWLEDGEMENTS}

The author acknowledges technical support and experimental setup by Mr. Steve Allman, Oak Ridge National Laboratory.

Oak Ridge National Laboratory is managed by UT-BATTELLE, LLC for the U.S. Department of Energy under contract DE-AC05-000R22725.

This report has been authored by a contractor of the U.S. Government under contract DEAC05-000R22725. Accordingly, the U.S. Government retains a nonexclusive, royalty-free license to publish or reproduce the published form of this contribution, or allow others to do so, for U.S. Government purposes. 


\section{REFERENCES}

1. Akishev, Y.S., A.A. Deryugin, I.V. Kochetov, A.P. Napartovich, and N.I. Trushkin (1993) DC Glow Discharge in Air Flow at Atmospheric Pressure Condition with Waste Gases Treatment, J. Phys. D: Appl. Phys., 26: 1630-1637.

2. Akishev, Y.S., A.A. Michail Grushin, A. Napartovich, and N.I. Trushkin (2002) Novel AC and DC Non-thermal Plasma Sources for Cold Surface Treatment of Polymer Films and Fabrics at Atmospheric Pressure, Plasmas and Polymers, 7: 261-289.

3. Asada, T., S. Yoshimune, and K. Yoshitaka (2002) Evaluation Method of Surface Cleanliness by Using Contact Angle Meter for UDI-H2O Cleaning, Kuki Seijo to Kontamineshyon Kontororu Kenkyu Taikai Yokoshu, 20: 176-178.

4. Cheng, M.-D., R. W. Smithwick, III, and R. Hinton (2006) Use of Electrically Enhanced Aerosol Plasma Spectroscopy for Real-Time Characterization of Beryllium Particles, JASTM Intern., 3(1): 1-10, JAI13172.

5. Ganguli, A. and R.D. Tarey (2002) Understanding Plasma Sources, Current Sci., 83: 279290.

6. Guo, Y.-B., F.C.-N. Hong (2003) Radio-Frequency Microdischarge Arrays for Large-Area Cold Atmospheric Plasma Generation, Appl. Phys. Lett., 82:337-339.

7. Haddock, R., B. Schwartz, S. Westwood (2003) Test Report for the Large-Frame Aircraft Decontamination Demonstration - Material Compatibility Test, DTRA test report \# 8-CO290-000-002.

8. Herrmann, H.W., I. Henins, J. Park, and G.S. Selwyn (1999) Decontamination of Chemical and Biological Warfare (CBW) Agents Using an Atmospheric Pressure Plasma Jet (APPJ), Phys. Plasma, 6:2284-2289.

9. Hudson, D.M. (1999) The Use of Contact Angle Analysis to Determine Surface Cleanliness, Metal Finishing, 95(10): 26-27.

10. Laroussi, M. (2002) Nonthermal Decontamination of Biological Media by AtmosphericPressure Plasmas: Review, Analysis, And Prospects, IEEE Trans. Plasma Sci., 30: 14091415.

11. Lee, D.-W. and M.-D. Cheng (2004a) Particle Generation by Laser Ablation during Surface Decontamination, J. Aerosol Sci., 35:1527-1540.

12. Lee, D.-W. and M.-D. Cheng (2004b) Investigation of Nanoparticle Generation during Surface Decontamination by Laser Ablation at Low Fluence, J. Aerosol Sci., 35:15131526.

13. Lin, Y. L. and M.-D. Cheng (2002) Removal of Contaminants from Target Surfaces by Laser Ablation: Formation of Nanoparticles, Presented at the 2002 National Meeting of American Association for Aerosol Research, Charlotte, NC, October.

14. Lu, X.P. and M. Laroussi (2006a) Dynamics of an Atmospheric Pressure Plasma Plume Generated by Submicrosecond Voltage Pulses, J. Appl. Phys., 100:063302.

15. Lu, X.P. and M. Laroussi (2006b) Temporal and Spatial Emission Behavior of Homogeneous Dielectric Barrier Discharge Driven by Unipolar Sub-Microsecond Square Pulses, J. Phys. D: Appl. Phys., 39:1127-1131.

16. Machala, Z., E. Marode, C.O. Laux, and C.H. Kruger (2004) DC Glow Discharges in Atmospheric Pressure Air, J. Adv. Oxid. Technol., 7:133-137. 
17. Schulte, F., J. Lingott, U. Panne, and J. Kneipp (2008) Chemical Characterization and Classification of Pollen, Anal. Chem., 80:9551-9556.

18. Tendero, C., C. Tixier, P. Tristant, J. Desmaison, and P. Leprince (2006) Atmospheric Pressure Plasma: A Review, Spectrochimica Acta Part B, 61: 2-30.

19. Walsh, J.L., J.J. Shi, and M.G. Kong (2006) Contrasting Characteristics of Pulsed and Sinusoidal Cold Atmospheric Plasma Jet, Appl. Phys. Lett., 88: 171501-171503

20. White, M.L. (1970) Clean Surfaces: Their Preparation and Characterization for Interfacial Studies, Marcel Dekker, New York, p 361.

21. Yeong, J.Y., S.E. Babayan, V.J. Tu, J. Park, I. Henins, R.F. Hicks, and G.S. Selwyn (1998) Etching Materials with an Atmospheric-Pressure Plasma Jet, Plasma Source Sci. Technol., $7: 282-285$. 
THIS PAGE WAS INTENTIONALLY LEFT BLANK 
ORNL/TM-2011/155

\section{INTERNAL DISTRIBUTION}

1. April McMillian

2. Richard Stoudder

3. Gary Jacobs

4. Trent Nicholas

5. ORNL Office of Technical Information and Classification

\section{EXTERNAL DISTRIBUTION}

6 R. Schoske, U.S. Air Force Academy, Colorado Spring, C0. Email: richard.schoske@usafa.edu 


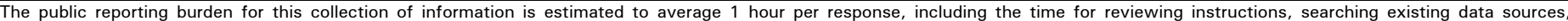

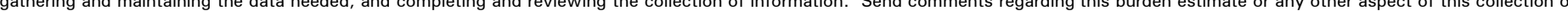

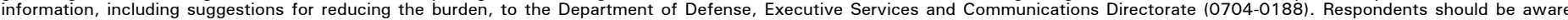

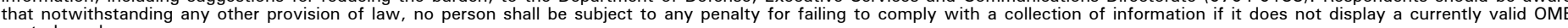
control number.

PLEASE DO NOT RETURN YOUR FORM TO THE ABOVE ORGANIZATION.
1. REPORT DATE (DD-MM- $Y Y Y Y)$
2. REPORT TYPE 30-06-2011
Project Final Report
3. DATES COVERED (From - To)
October 2009 - September 2011

\section{TITLE AND SUBTITLE}

Testing of Nonthermal Plasma for Decontamination on Sensitive

Weapons Systems and Platforms

5a. CONTRACT NUMBER
WP 1760

5b. GRANT NUMBER

$N / A$

5c. PROGRAM ELEMENT NUMBER

$\mathrm{N} / \mathrm{A}$

6. $A U T H O R(S)$

Meng-Dawn Cheng

$\mathrm{N} / \mathrm{A}$

5f. WORK UNIT NUMBER

ORNL Environmental Sciences Division (42)

7. PERFORMING ORGANIZATION NAME(S) AND ADDRESS(ES)

Oak Ridge National Laboratory

PO Box 2001

Oak Ridge, TN 37831-6038

9. SPONSORING/MONITORING AGENCY NAME(S) AND ADDRESS(ES)

Strategic Environmental Research and Development Program

901 N. Stuart Ave.,

Arlington, VA
8. PERFORMING ORGANIZATION REPORT NUMBER

ORNL/TM-2011/155

10. SPONSOR/MONITOR'S ACRONYM(S)

SERDP

11. SPONSOR/MONITOR'S REPORT NUMBER(S)

\section{DISTRIBUTION/AVAILABILITY STATEMENT}

The submitted manuscript has been authored by a contractor of the U.S. Government under contract

DE-AC05-00OR22725. Accordingly, the U.S. Government retains a nonexclusive, royalty-free license to publish or reproduce the published form of this contribution, or allow others to do so, for U.S. Government purposes.

\section{SUPPLEMENTARY NOTES}

$\mathrm{N} / \mathrm{A}$

\section{ABSTRACT}

Efficient decontamination of military platforms and systems represents the first line of defense and protection for U.S. warfighters. Using solvents, wet decontamination approach generates secondary pollution and requires extra care of the contaminated solutions afterwards. The wet approach could also degrade material integrity of the treated platforms and systems. Alternative decontamination technique that does not erode the integrity and not produce secondary contamination is required. Nonthermal plasma was tested as a decontamination alternative for a number of pollutants in this project. A radiofrequency-powered nonthermal plasma technique was applied to biological aerosols, oil and grease, and paint-stained airframe structural material. Raman spectroscopy, scanning electron microscopy, and aerosol monitoring techniques were used to assess the effectiveness of the plasma decontamination technique. The nonthermal plasma technique was found to be useful in the cleanup of the stained surface, reduce the potency of the biological agents, and maintain the surface structure intact. Time-sequenced Raman analysis on biological aerosols indicates the

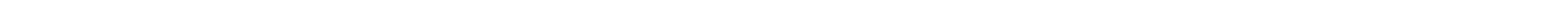

15. SUBJECT TERMS

Nonthermal Plasma, Contaminants, Decontamination, Chemical and Biological Agents

16. SECURITY CLASSIFICATION OF: \begin{tabular}{|l|l|l|}
\hline a. REPORT & b. ABSTRACT & c. THIS PAGE \\
\hline
\end{tabular}

\section{LIMITATION OF ABSTRACT}

None
18. NUMBER OF PAGES 34 19a. NAME OF RESPONSIBLE PERSON Meng-Dawn Cheng 19b. TELEPHONE NUMBER (Include area code) 865-241-5918 